\title{
REMARKS ON A PAPER BY A. AZIZ
}

\section{ZALMAN RUBINSTEIN}

\begin{abstract}
The paper consists of two parts. In the first part a short proof of the main theorem due to A. Aziz on the location of zeros of composite polynomials is given. In the second part some properties of a fixed length continuation of a polynomial are deduced.
\end{abstract}

In this note we shall indicate a short proof of the main theorem in [1] concerning the zeros of composite polynomials and then apply these results to consider a problem in fixed length continuation of polynomials.

1. The following main theorem was proved in [1] via several lemmas and Grace's theorem.

THEOREM A. If $P(z)=\sum_{j=0}^{n} C(n, j) A_{j} z^{j}$ and $Q(z)=\sum_{j=0}^{m} C(m, j) B_{j} z^{j}$ are two polynomials of degree $n$ and $m$ respectively, $m \leqslant n$, such that

$$
C(m, 0) A_{0} B_{m}-C(m, 1) A_{1} B_{m-1}+\cdots+(-1)^{m} C(m, m) A_{m} B_{0}=0,
$$

then the following holds:

(i) If $Q(z)$ has all its zeros in the disk $|z| \leqslant r$, then $P(z)$ has at least one zero in $|z| \leqslant r$.

(ii) If $P(z)$ has all its zeros in the region $|z| \geqslant r$, then $Q(z)$ has at least one zero in $|z| \geqslant r$.

We propose the following short

PROOF. (i) Relation (1) is invariant under the transformation $z \rightarrow r z$ in $P(z)$ and $Q(z)$ so that we may assume $r=1$. Assume by contradiction that all zeros of $P(z)$ lie in $|z|>1$. By the well known Gauss-Lucas result on the zeros of the derivative of a polynomial, all the zeros of

$$
P_{1}(z)=D^{(n-m)}\left[z^{n} P(1 / z)\right]=n(n-1) \cdots(m+1) \sum_{j=0}^{m} C(m, j) A_{j} z^{m-j}
$$

lie in $|z| \leqslant 1$, so that the zeros of

$$
P_{2}(z)=z^{m} P_{1}(1 / z)=\sum_{j=0}^{m} C(m, j) A_{j} z^{j}
$$

lie in $|z|>1$.

Received by the editors May 22, 1984.

1980 Mathematics Subject Classification. Primary 30C15; Secondary 30C10.

\footnotetext{
1985 American Mathematical Society $0002-9939 / 85 \$ 1.00+\$ .25$ per page
} 
The hypothesis (1) means that $P_{2}(z)$ and $Q(z)$ are apolar, so that by Grace's theorem [3, Theorem 15.3] the region $|z|>1$ in the extended complex plane which contains all the zeros of $P_{2}(z)$ contains at least one zero of $Q(z)$. If the degree of $P_{2}$ is $m-k$, we shall assume that $P_{2}$ has $k$ zeros at the point at infinity so that the total number of zeros of $P_{2}$ is $m$. This is a contradiction to the hypothesis on the zeros of $Q(z)$.

Actually, one can avoid using the extended complex plane by assuming first that $A_{m} \neq 0$, then applying a continuity argument.

Proof of assertion (ii) is similar to the proof of (i). Since the zeros of $P(z)$ all lie in $|z| \geqslant 1$, so do the zeros of $P_{2}(z)$. The conclusion follows by Grace's theorem.

2. Let $m$ and $n$ be fixed integers, $1 \leqslant m \leqslant n$, and let $p(z)=\sum_{j=0}^{m} C(n, j) A_{j} z^{j}$, $A_{0}=1, A_{m} \neq 0$, be a fixed polynomial. Any polynomial $q(z)$ of the form

$$
q(z)=p(z)+a_{m+1} z^{m+1}+\cdots+a_{n} z^{n}
$$

will be called a continuation of $p(z)$ of length $n$. Denote the family of all such continuations by $\Pi(p, n)$. It is well known [2] that for sufficiently large $n$ the zeros of polynomials in $\Pi$ can be made to lie on an arbitrary Jordan curve which contains the origin in its interior. However, if, as we assumed, $n$ is fixed, there exists a largest disk about the origin free of zeros of all members of $\Pi$. We shall give an estimate of the radius of this disk and formulate a conjecture with regards to its exact value.

For each $q \in \Pi$ let $\mu(q)=\min _{1 \leqslant k \leqslant n}\left|z_{k}\right|$, where the $z_{k}$ are the zeros of $q$ with the convention that $q$ has $(n-\operatorname{deg} q)$ zeros at the point at infinity. We estimate $\rho=\rho(p, n)$ defined by

$$
\rho=\sup _{q \in \mathrm{II}} \mu(q) .
$$

$A$ priori it is not clear that $\rho$ is finite. If $A_{s}$ is the first nonzero coefficient with $s \geqslant 1$, then it follows by a theorem of Van Vleck [3, Theorem 33.3] that every $q \in \Pi$ has a zero in the disk $|z| \leqslant\left[C(n, s) /\left|A_{s}\right|\right]^{1 / s}$. Thus, $0<\rho<\infty$.

THEOREM. If $\rho$ is defined by (2), then the inequality

$$
\rho \leqslant \mu\left(\sum_{j=0}^{m} C(m, j) A_{j} B_{j} z^{j}\right) / \mu\left(\sum_{j=0}^{m} C(m, j) B_{j} z^{j}\right)
$$

holds for any choice of complex numbers $B_{0}, B_{1}, \ldots, B_{m}, B_{0} B_{m} \neq 0$.

For the proof of the theorem we shall need this corollary of Theorem A ([1, Theorem 2] or [3, Theorem 16.1]).

THEOREM B. If all the zeros of $P(z)=\sum_{j=0}^{n} C(n, j) A_{j} z^{j}$ of degree $n$ lie in $|z| \geqslant r$ and if $Q(z)=\sum_{j=0}^{m} C(m, j) B_{j} z^{j}, B_{0} B_{m} \neq 0, m \leqslant n$, then every zero $\omega$ of the polynomial $R(z)=\sum_{j=0}^{m} C(m, j) A_{j} B_{j} z^{j}$ has the form $\omega=-\alpha \beta$ where $\beta$ is a zero of $Q$ and $|\alpha| \geqslant r$.

Proof of The theorem. Fix $B_{j}, j=0,1, \ldots, m, B_{0} B_{m} \neq 0$. Let $P^{*} \in \Pi$ such that $\mu\left(P^{*}\right) \geqslant \rho-\varepsilon$. We may assume that $P^{*}=P$ of Theorem B and $r=\rho-\varepsilon$. By Theorem $\mathrm{B}, \mu(R) \geqslant(\rho-\varepsilon)|\beta|$, where $\beta$ is a zero of $Q$. Thus, $\mu(R) \geqslant(\rho-\varepsilon) \mu(Q)$ and letting $\varepsilon \rightarrow 0, \mu(R) \geqslant \rho \mu(Q)$. This completes the proof. 
One notices that the existence of an extremal polynomial $P^{*} \in \Pi$ for which $\mu\left(P^{*}\right)=\rho$ was not assumed. Indeed, it does not seem simple to answer the question of whether such a polynomial exists for every given $P$. Choosing $B_{j}=1$ in (3) and noticing that $\sum_{j=0}^{m} C(n, j) A_{j} z^{j} \in \Pi$ we have

Corollary. If $A_{m} \neq 0$, then

$$
\mu\left(\sum_{j=0}^{m} C(n, j) A_{j} z^{j}\right) \leqslant \rho \leqslant \mu\left(\sum_{j=0}^{m} C(m, j) A_{j} z^{j}\right) .
$$

Although we have assumed that $A_{0} \neq 0$, it is easy to see that the last inequalities hold as equalities if $A_{0}=0$. Since the $A_{j}$ are arbitrary subject to $A_{m} \neq 0$, the corollary shows that, for a given polynomial $P$ of degree $m$, there are multiplier sequences idependent of the coefficients which increase (decrease) $\mu(P)$. Their study may be worthwhile but it is much beyond the scope of this note.

We conclude with a

Conjecture. There exists an extremal polynomial $P^{*} \in \Pi$ for which $\mu\left(P^{*}\right)=\rho=$ inf $f\left(B_{0}, B_{1}, \ldots, B_{m}\right)$ where $f\left(B_{0}, B_{1}, \ldots, B_{m}\right)$ denotes the right-hand side of inequality (3).

This conjecture is true for $m=1$ and arbitrary $n$. Indeed, one verifies that for $p(z)=1+a z, \rho=n /|a|$, an extremal polynomial is $(1+(a / n) z)^{n}, A_{0}=1, A_{1}=$ $a / n$ so that we have equality in (3).

\section{REFERENCES}

1. Abdul Aziz, On the zeros of composite polynomials, Pacific J. Math. 103 (1982), 1-7.

2. L. I. Gavrilov, On the K-continuation of polynomials, Mat. Sb. 36 (78) (1955), 271-274.

3. M. Marden, Geometry of polynomials, Math. Surveys, No. 3, Amer. Math. Soc., Providence, R. I., 1966.

Department of Mathematics, University of Haifa, Mount Carmel, Haifa, Israel

Current address: Department of Mathematics, University of Colorado, Boulder, Colorado 80309 